\title{
Experimental observation of electrical instability of droplets on dielectric layer
}

\author{
Jiang-Tao Feng and Ya-Pu Zhao ${ }^{1}$ \\ State Key Laboratory of Nonlinear Mechanics, Institute of Mechanics, Chinese Academy of Sciences, \\ Beijing 100080, People's Republic of China \\ E-mail: yzhao@imech.ac.cn
}

Received 10 January 2008, in final form 21 January 2008

Published 11 February 2008

Online at stacks.iop.org/JPhysD/41/052004

\begin{abstract}
Polydimethylsiloxane (PDMS) has become the most widely used silicon-based organic polymer in bio-MEMS/NEMS devices. However, the inherent hydrophobic nature of PDMS hinders its wide applications in bio-MEMS/NEMS for efficient transport of liquids.

Electrowetting is a useful tool to reduce the apparent contact angle of partially wetting conductive liquids and has been utilized widely in bio-MEMS/NEMS. Our experimental results show that the thin PDMS membranes exhibit good properties in electrowetting-on-dielectric. The electrical instability phenomenon of droplets was observed in our experiment. The sessile droplet lying on the PDMS membrane will lose its stability with the touch of the wire electrode to make the apparent contact angle change suddenly larger than $35^{\circ}$. Contact mode can protect the dielectric layer from electrical breakdown effectively.

Electrical breakdown process of dielectric layer was recorded by a high speed camera. It is found experimentally that a PDMS membrane of $4.8 \mu \mathrm{m}$ thick will not be destroyed due to the electric breakdown even at $800 \mathrm{~V}$ in the contact mode.
\end{abstract}

\section{Introduction}

In 1879 Lord Rayleigh [1] found that an electrically charged droplet becomes unstable when the outward electrostatic forces balance the surface tension. Lippmann's famous experiment in 1875 verified how the surface tension of mercury is altered by the application of an electrical potential. As a matter of fact, Lippmann's 1875 paper [2] provided the starting point for electrowetting (EW) experiments. As a basic equation for EW, Lippmann's equation presented in that 1875 paper considers the reduction in solid-liquid surface tension as a function of applied voltage, dielectric layer thickness and the dielectric constant. EW is a useful tool for manipulating liquid droplets and has aroused great interest in recent years as it has found wide applications in bio-MEMS/NEMS. The manipulations of the microfluidics have attracted increasing attention [3-10]. The classic effect of

\footnotetext{
1 Author to whom any correspondence should be addressed.
}

electrowetting-on-dielectric (EWOD) is making the apparent contact angle decrease by applying voltages between the electrodes in the droplet and the counter electrode [11]. The most common configuration for experiments and practical applications in EW is that a typical dielectric layer (insulator film) of micrometre thickness separates the metal electrode from the conductive droplet [12-16]. This experimental method is called EWOD. In the current research about EWOD, the phenomena on different dielectric materials in microdevices are studied [17-21]. Several fundamental limitations in EW have been studied by Vallet et al [22].

Polydimethylsiloxane (PDMS) elastomer has become one of the most widely used materials for various microfluidic devices, rapid prototyping and nanolithography [23, 24]. The advantages of PDMS include cheapness, optical transparency, simple fabrication, good biocompatibility, chemically inertness and non-toxicity. It has been applied to many bio-MEMS/NEMS and other fields as substrate and microfluidic devices. However, the hydrophobic 

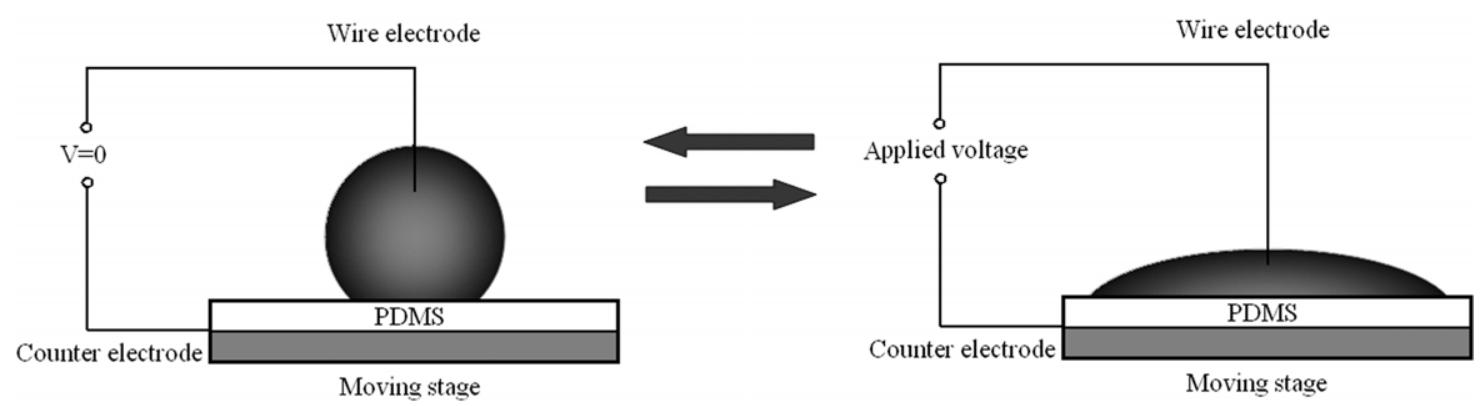

Figure 1. Sketch of EW setup including wire electrode, counter electrode on a moving stage covered with PDMS membrane, dc electrical resource and conductive droplet. The external voltage is applied between a thin Pt wire electrode and counter electrode.

nature of PDMS limits its applicability for microfluidic patterning and other applications in bio-MEMS/NEMS. Many techniques have been utilized to generate hydrophilic PDMS surface, including physical methods, chemical methods or a combination of both [25-30]. Nevertheless, these techniques do not meet the demands, due to the quick hydrophobic recovery, the complicated process or the chemical contaminant. EW is an effective way to hydrophilize a PDMS membrane [31].

In this paper, PDMS membrane properties as a dielectric layer were studied experimentally. Systematic experimental results show that PDMS exhibits good properties as a dielectric layer. A moving stage was used to change the electrodemembrane distance to realize finite function. The instability process of droplets was observed which can reduce the apparent contact angle larger than $35^{\circ}$.

\section{Experiment setup}

The sketch of our experimental setup is shown in figure 1 . It contains a wire electrode, a counter electrode on a moving stage covered with PDMS membrane, a dc electrical resource, a conductive droplet and an OCA20 system to measure the contact angle. The droplet displays Young's contact angle at zero voltage (figure 1, left part) and a reduced (LippmannYoung) contact angle at finite voltage (figure 1, right part). The moving stage can be moved to change the electrodemembrane distance during our experiment. When an external electrical potential is applied between the two electrodes, the charges redistribute and modify the apparent contact angle.

\section{Material preparation}

The PDMS (polydimethylsiloxane, Sylgard 184, Dow Corning, USA; ratio of the base to curing agent $=10: 1$ ) which we used was vacuumed for an hour to remove the trapped air-bubbles.

The PDMS films were spin coated on the indium-tin-oxide (ITO) conductive glass with a size of $40 \mathrm{~mm} \times 40 \mathrm{~mm} \times 1.1 \mathrm{~mm}$. Films of different thicknesses were obtained by varying the spin speed of the spin coater (KW-4A). The relationship between the spin speed and thickness of PDMS has been obtained by Dai and Zhao [31] and is shown in figure 2. Then the PDMS films were dried in air at $80^{\circ} \mathrm{C}$ for $4 \mathrm{~h}$. In our

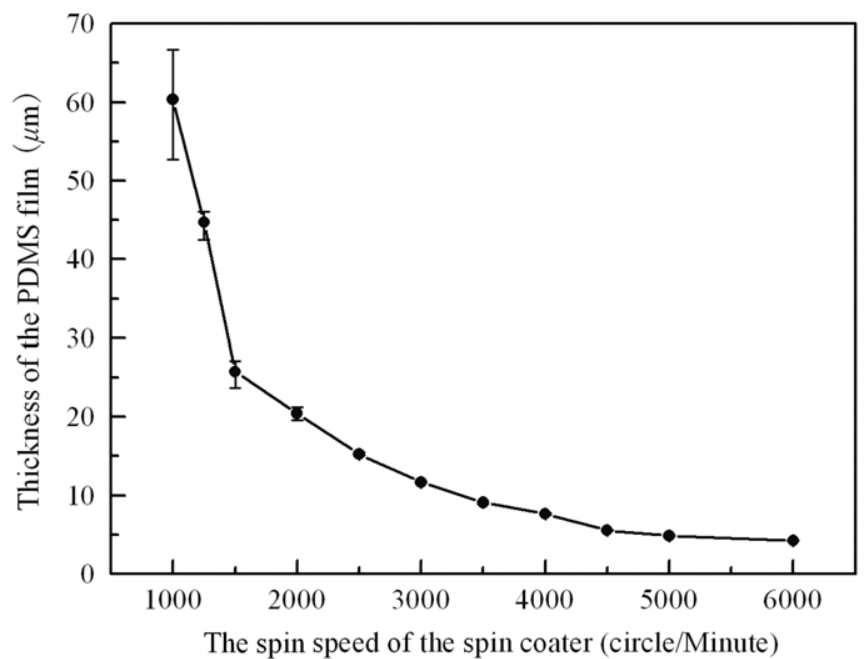

Figure 2. Relationship between PDMS film thickness and the rotating speed of the spin coater.

experiment, the ITO layer was used as the counter electrode. Several pieces of ITO glass have been cleaned by sonicleaning for three periods of $5 \mathrm{~min}$ using high purity water, reagent acetone and ethanol as detergents, respectively.

Contact angles were measured with the OCA20 system (precision $\pm 0.1^{\circ}$ ) from Dataphysics, Germany. A sessile drop method was used to determine the contact angle of water on different surfaces. The conductive liquid we used is $\mathrm{KCl}$ solution $\left(0.1 \mathrm{moll}^{-1}\right)$. The volume of the droplet is $3 \mu \mathrm{l}$. Under this condition, the Bond number

$$
B o=\sqrt{\frac{g \rho d^{2}}{4 \gamma_{\mathrm{lv}}}} \approx 0.37,
$$

which compares the gravity and surface tension, is smaller than unity (gravity acceleration: $g=9.8 \mathrm{~m} \mathrm{~s}^{-2}$, droplet diameter: $d \cong 2 \times 10^{-3} \mathrm{~m}$, interface tension: $\gamma_{\mathrm{lv}}=72 \times 10^{-3} \mathrm{~N} \mathrm{~m}^{-1}$, mass density: $\rho \approx 10^{3} \mathrm{~kg} \mathrm{~m}^{-3}$, here surface energy and density of water in $25^{\circ}$ were used). So the influence of gravity is neglected in this paper.

\section{Instability process in contact mode}

The distance between the wire electrode and the PDMS membrane (electrode-membrane distance) will influence the 


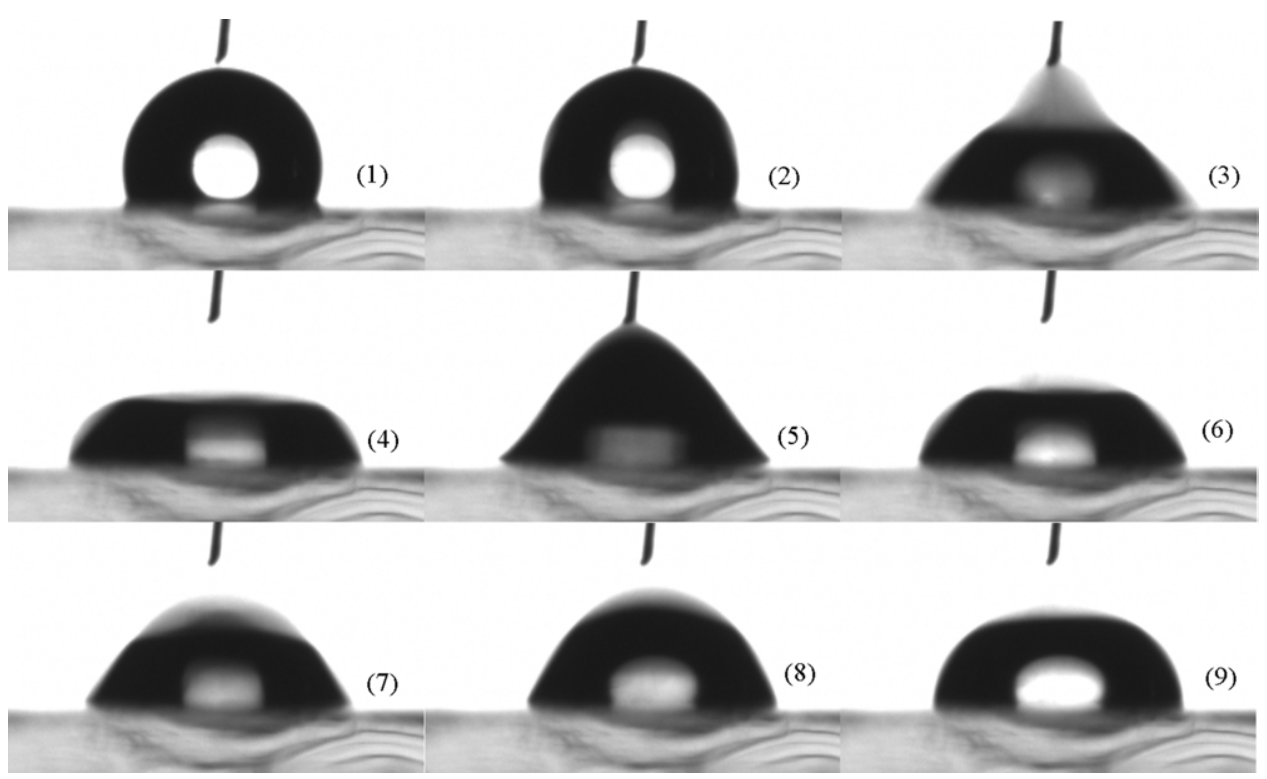

Figure 3. Instability process of first contact.

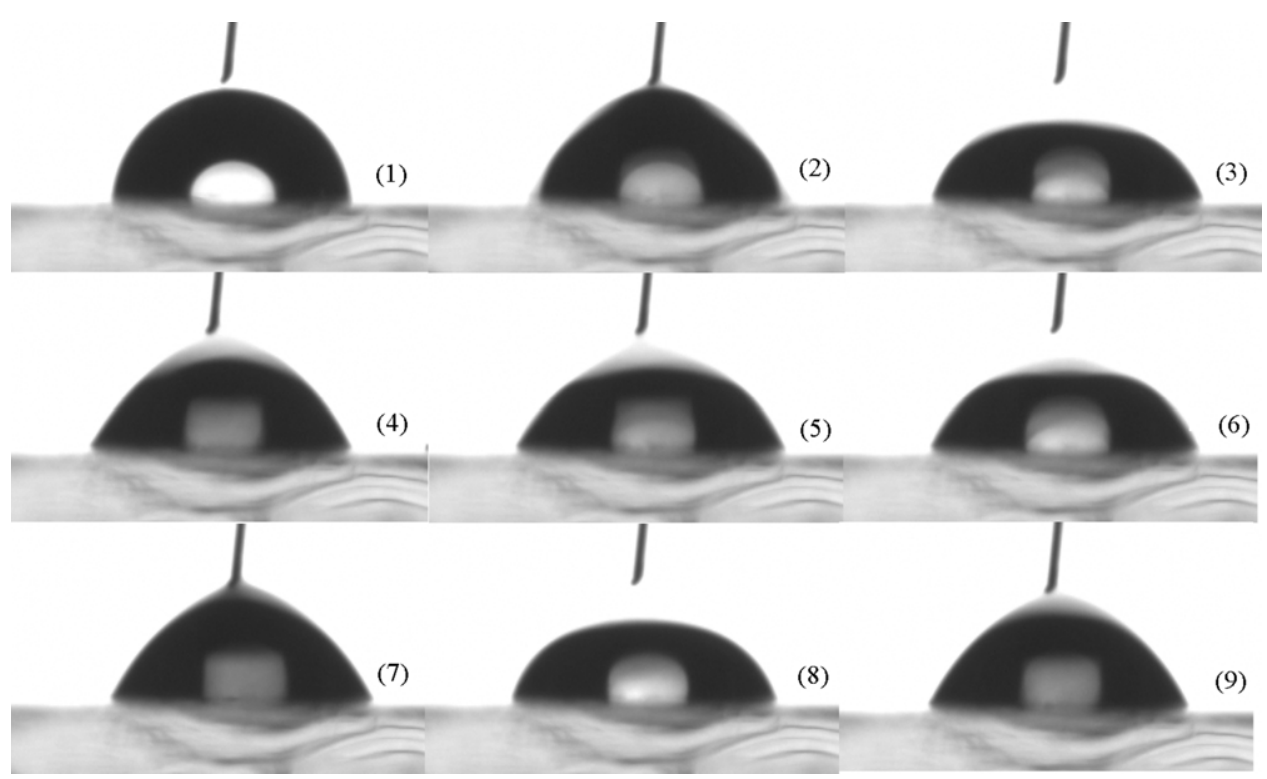

Figure 4. Instability process of second contact.

EW behaviours of the PDMS membrane. The PDMS membrane (spin speed: $4000 \mathrm{r} \mathrm{min}^{-1}$, thickness: $7.6 \mu \mathrm{m}$ ) was used as an example to explain the instability process in our experiment. For simplicity, the whole process was separated into four steps which are shown in figure 3 :

- Step 1. Withdraw the wire electrode to remove it from the droplet. Then the voltage of dc electrical source was increased to $350 \mathrm{~V}$.

- Step 2. The electrode-membrane distance is reduced to make the conductive droplet contact with the wire electrode. The contact angle of the droplet jumps from $108.7^{\circ}$ to $86.1^{\circ}$. This is an unstable process and its characteristic time is of the order $10 \mathrm{~ms}$. A video sequence of this process is shown in figure 3 , and the interval between each picture is $5 \mathrm{~ms}$. The droplet will oscillate in its natural frequency of about $100 \mathrm{~Hz}$. In fact, the unstable process starts when the wire electrode is still at a short distance from the droplet. This situation may be caused by the ionization of air in the vicinity of the top end of the wire electrode. The ionization of air causes the wire electrode to discharge and the droplet receives charges.

- Step 3. Lift the moving stage to make the droplet contact with the wire electrode again. The contact angle of the droplet jumps from $86.6^{\circ}$ to $79.0^{\circ}$. This is also an unstable process. A video sequence of this process is shown in figure 4 , and the interval between each picture is $5 \mathrm{~ms}$. The droplet will oscillate in its natural frequency of about $100 \mathrm{~Hz}$. The stable situations of steps 3 and 4 after oscillation can be shown as figure 5 . The former three steps are called contact mode EW. 


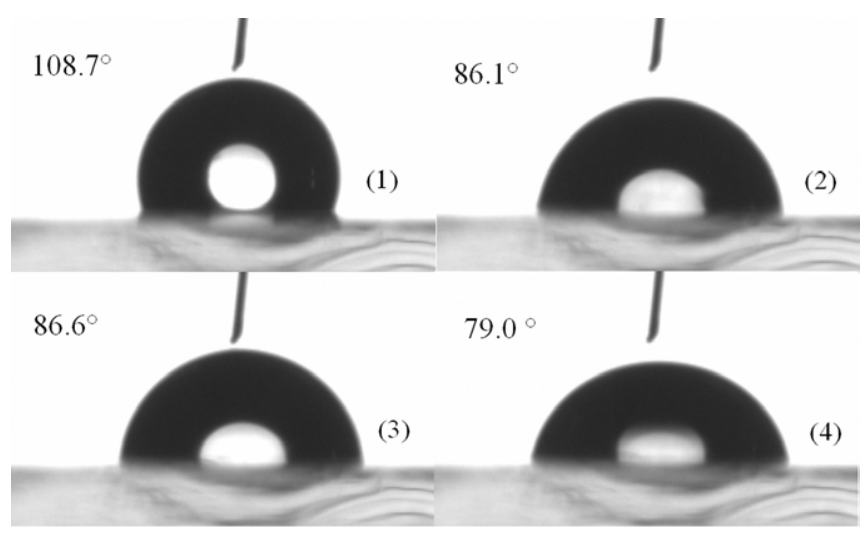

Figure 5. Whole instability process. Contact angle: (1) $108.7^{\circ}$, (2) $86.1^{\circ}$, (3) $86.6^{\circ}$ and (4) $79.0^{\circ}$.

- Step 4. The electrode-membrane distance is reduced further to make the wire electrode insert the droplet. The contact angle decreases from $79.2^{\circ}$ to $61.8^{\circ}$. Occasionally there is only one instability step in our experiment.

\section{Estimation of instability time in contact mode}

In our experiment, the characteristic time of the unstable process when the wire electrode contacts with the droplet in contact mode is found to be of the order of $10 \mathrm{~ms}$. We used the theory of Lord Rayleigh [32] to estimate it. The period of a free droplet in free oscillation is

$$
T=\frac{\pi}{4} \sqrt{\frac{\rho d^{3}}{\gamma_{\mathrm{lv}}}} \sim 8.3 \times 10^{-3} \mathrm{~s},
$$

where $d \cong 2 \times 10^{-3} \mathrm{~m}, \gamma_{\mathrm{lv}}=72 \times 10^{-3} \mathrm{Nm}^{-1}, \rho \approx$ $10^{3} \mathrm{~kg} \mathrm{~m}^{-3}$ (here surface energy and density of water at $25^{\circ} \mathrm{C}$ were used). The theoretical result in equation (2) is found on the same order with the experimental observation. This is just an approximate result and there may be some errors in the method such as:

- The droplet in partial wetting is not the same with that of whole droplet in air.

- The surface energy and density of water are not the same as that of the $\mathrm{KCl}$ solution $\left(0.1 \mathrm{moll}^{-1}\right)$.

\section{Electrical breakdown of dielectric layer}

Electrical breakdown of dielectric layer will happen when the voltage is high enough [18]. Many factors, including dielectric layer thickness, contact time between wire electrode and droplet, electrode-membrane distance and physical properties of dielectric layer, etc, can influence the critical voltage of breakdown. It is pointed out by Moon et al [18] that critical voltage of breakdown is proportional to the thickness of the Telflon AF layer. The relationship of critical voltage of breakdown and thickness of PDMS film is shown in table 1. Table 1 shows that the critical voltage of breakdown
Table 1. Relationship among spin speed (rotations $\mathrm{min}^{-1}$ ), thickness of PDMS membrane and breakdown voltage.

\begin{tabular}{lll}
\hline $\begin{array}{l}\text { Spin speed } \\
\left(\text { rotations } \mathrm{min}^{-1}\right)\end{array}$ & $\begin{array}{l}\text { Thickness } \\
(\mu \mathrm{m})\end{array}$ & $\begin{array}{l}\text { Breakdown } \\
\text { voltage }(\mathrm{V})\end{array}$ \\
\hline 3000 & 11.7 & 664 \\
4000 & 7.6 & 320 \\
5000 & 4.8 & 228 \\
\hline
\end{tabular}

increases with the increase in PDMS film thickness. The whole process of breakdown which was recorded by a high speed camera in $200 \mathrm{~Hz}$ is shown in figure 6. The whole process is about $30 \mathrm{~ms}$. The interval between each picture is $5 \mathrm{~ms}$. A sparkle can be seen in figure 6(2) and this is the start of breakdown. In this stage, the dielectric layer was destroyed and there is instant current between wire and counter electrodes. The heat generated by the instant current makes the bubbles generated by electrolysis of electrolyte swell dramatically, like an explosion. In this process, electrical energy was turned to dynamic energy of conductive liquids and sputtered spray can be seen in figures 6(4-6). Bubbles can still always be observed after breakdown, such as in figure 7(4).

By our contact mode of EW, the electrode-membrane distance was the largest distance to generate the EW effect and contact time between wire electrode and droplet was very short-less than $5 \mathrm{~ms}$. This will dramatically lower the chance of breakdown. Even voltage higher than $800 \mathrm{~V}$ (the upper limit of our electrical source) cannot induce breakdown in the PDMS membrane (spin speed: $5000 \mathrm{r} \mathrm{min}^{-1}$, thickness: $4.8 \mu \mathrm{m}$ ) during the former two steps while in normal mode the average critical voltage of a PDMS membrane with the same thickness is $228 \mathrm{~V}$. Figure 7 shows the situation of EW on the PDMS membrane (voltage: $800 \mathrm{~V}$, spin speed: 5000 rotations $\mathrm{min}^{-1}$, thickness: $\left.4.8 \mu \mathrm{m}\right)$. In step 2 (figure $7(2)$ ), the contact angle changes from $112.8^{\circ}$ to $85.9^{\circ}$. In step 3 (figure 7(3)), the contact angle changes from $86.2^{\circ}$ to $74.2^{\circ}$. In step 4 , bubbles can be seen in the droplet and this means that dielectric layer has been destroyed due to electrical breakdown. The contact angle is $76.7^{\circ}$ in this step.

\section{Conclusions}

The influence of electrode-membrane distance on EW behaviours of PDMS was studied in this paper. The contact mode EW can protect the dielectric layer from breakdown effectively. The wire electrode just contacts the droplet without inserting it to decrease the chance of contaminant. The breakdown of the PDMS membrane was recorded by a high speed camera and PDMS fragments and sputtered sprays can be observed in figures 6(4-6). The electric instability process can cause the droplet flatten out and change the contact angle larger than $30^{\circ}$.

\section{Acknowledgments}

This work was jointly supported by the National Basic Research Program of China (973 Program, Grant 


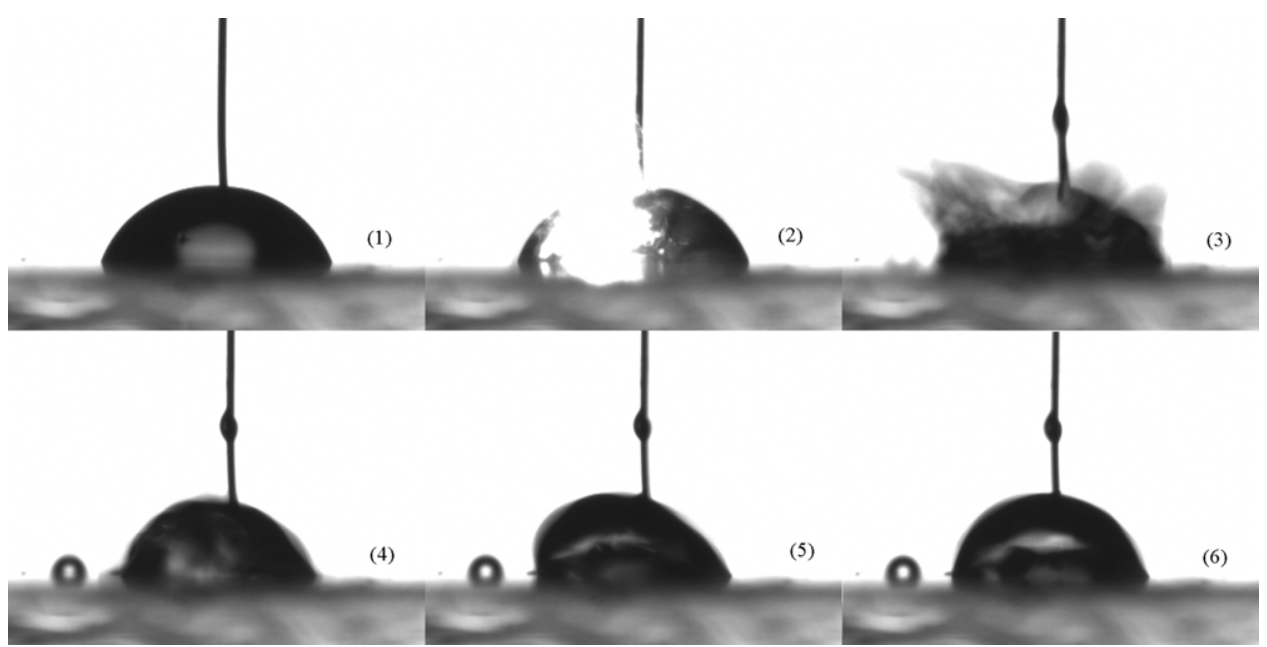

Figure 6. Breakdown process.

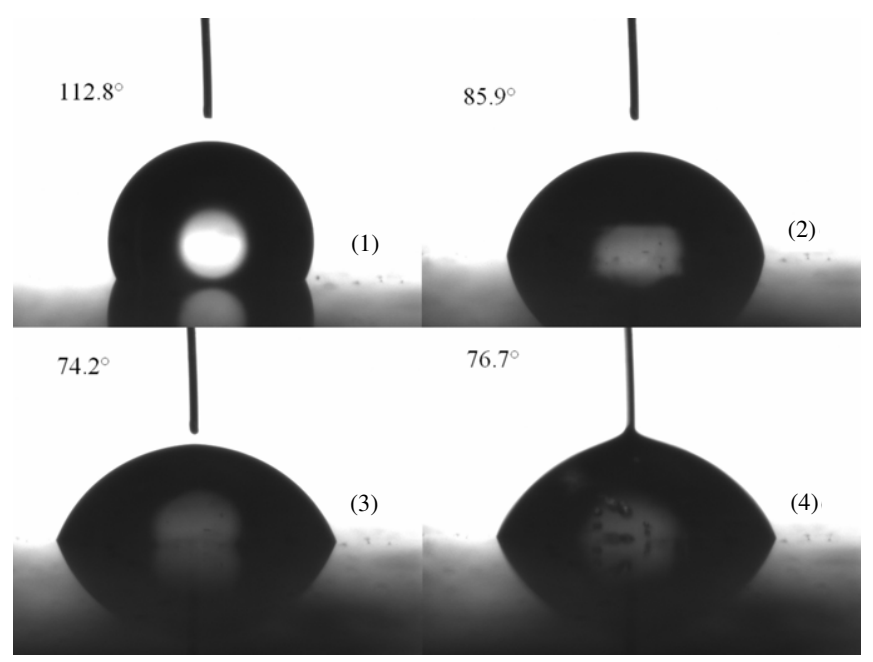

Figure 7. Contact mode (voltage $800 \mathrm{~V}$, spin speed $5000 \mathrm{r} \mathrm{min}^{-1}$, thickness $4.8 \mu \mathrm{m})$.

No 2007CB310500) and National Natural Science Foundation of China (NSFC, Grant No 10772180).

\section{References}

[1] Lord Rayleigh 1879 Proc. R. Soc. 2971

[2] Lippmann G 1875 Ann. Chim. Phys. 5494

[3] Cho S K, Moon H and Kim C J 2003 J. Microelectromech. Syst. 1270

[4] Velev O D, Prevo B G and Bhatt K H 2003 Nature 42515

[5] Yun K S and Yoon E 2005 Biomed. Microdevices 735

[6] Paik P, Pamula V K, Pollack M G and Fair R B 2003 Lab Chip 328

[7] Lee J and Kim C J 2000 J. Microelectromech. Syst. 9171
[8] Pollack M G, Fair R B and Shenderov A D 2000 Appl. Phys. Lett. 771725

[9] Lee J, Moon H, Fowler J, Schoellhammer T and Kim C J 2002 Sensors Actuators A $\mathbf{9 5} 259$

[10] Yoon J Y and Garrell R L 2003 Anal. Chem. 755097

[11] Mugele F, Klingner A, Buehrle J, Steinhauser D and Herminghaus S 2005 J. Phys.: Condens. Matter 17 S559

[12] Quilliet C and Berge B 2001 Curr. Opin. Colloid Interface Sci. 634

[13] Cheng J Y and Hsiung L C 2004 Biomed. Microdevices 6341

[14] Jones T B 2005 J. Micromech. Microeng. 151184

[15] Quinn A, Sedev R and Ralston J 2005 J. Phys. Chem. B 1096268

[16] Mugele F and Baret J C 2005 J. Phys.: Condens. Matter 17 R705

[17] Welters W J J and Fokkink L G J 1998 Langmuir 141535

[18] Moon H, Cho S K, Garrell R L and Kim C J 2002 J. Appl. Phys. 924080

[19] Berry S, Kedzierski J and Abedian B 2006 J. Colloid Interface Sci. $\mathbf{3 0 3} 517$

[20] Seyrat E and Hayes R A 2001 J. Appl. Phys. 901383

[21] Verheijen H J J and Prins M W J 1999 Langmuir 156616

[22] Vallet M, Vallade M and Berge B 1999 Eur. Phys. J. B 11583

[23] McDonald J C and Whitesides G M 2002 Accounts Chem. Res. 35491

[24] Fujii T 2002 Microelectron. Eng. 61-62 907

[25] Seo J and Lee L P 2006 Sensors Actuators B 119192

[26] Hillborg H, Sandelin M and Gedde U M 2001 Polymer 427349

[27] Hillborg H, Ankner J F, Gedde U M, Smith G D, Yasuda H K and Wikstrom K 2000 Polymer 416851

[28] Hillborg H and Gedde U M 1998 Polymer 391991

[29] Bodas D and Khan-Malek C 2006 Microelectron. Eng. 841277

[30] Feng J T and Zhao Y P 2008 Biomed. Microdevices 1065

[31] Dai W and Zhao Y P 2007 Int. J. Nonlinear Sci. Numer. Simul. 8519

[32] Lord Rayleigh 1877 The Theory of Sound 1st edn (London: Macmillan) 